\title{
Effets de la cyanamide hydrogène et de l'acide gibbérellique sur la levée de dormance du pommier 'Dorsett Golden' au sud du Maroc
}

Ahmed Mahhou*, Hassan Alahoui, Rachida Jadari

Département d'Horticulture, Institut Agronomique et Vétérinaire Hassan II, BP 6202-Instituts, Rabat, Maroc

a.mahhou@iav.ac.ma
${ }^{*}$ Correspondance et tirés à part

Fruits, 2003, vol. 58, p. 229-238 (C) 2003 Cirad/EDP Sciences All rights reserved

DOI: $10.1051 /$ fruits:2003011

RESUMEN ESPAÑOL, p. 238

\section{Effects of hydrogen cyanamide and gibberellic acid on the bud break of the 'Dorsett Golden' apple trees in Southern Morocco.}

Abstract - Introduction. The lack of chilling in Moroccan valleys constitutes a limiting factor for apple production. We evaluated the effects of hydrogen cyanamide (Dormex), alone or associated with mineral oil, and gibberellic acid on bud break of apple trees, under the climatic conditions of plains in Southern Morocco. Materials and methods. The experiments were conducted on 'Dorsett Golden' variety, known for its low chilling requirements. A randomised complete block design with four repetitions was used. The chemicals tested were Dormex, applied either alone at $(1.5,2.5$ and 4$) \%$ of the formulated product, or at $0.5 \%$ in association with mineral oil at (2 or 4)\%, and gibberellic acid at $460 \mathrm{mg} \cdot \mathrm{L}^{-1}$. The control trees were sprayed with water. The parameters evaluated were: bud break, flowering, fruit set, vegetative growth and production. Results. The chemicals advanced the date of bud break, improved its rate and that of flowering, and enhanced vegetative growth. The fruit yield and size were also significantly improved. Conclusion. Dormex, alone or combined with mineral oil and gibberellic acid, advanced and grouped the bud break and improved the fruit yield of the 'Dorsett Golden' apple trees. The effectiveness of the mixture [Dormex + mineral oil] associated with its lower cost make of this treatment a potential solution for the lack of chilling in the Moroccan areas characterized by mild winters.

Morocco / Malus / dormancy breaking / cold / cyanamide / gibberellic acid / flowering / fruiting / yields

\section{Effets de la cyanamide hydrogène et de l'acide gibbérellique sur la levée de dormance du pommier 'Dorsett Golden' au sud du Maroc.}

Résumé - Introduction. L'insuffisance en froid hivernal dans les zones de plaine au Maroc constitue un facteur limitant de la production du pommier. Nous avons évalué les effets du cyanamide hydrogène (Dormex), seul ou additionné d'huile minérale, et de l'acide gibbérellique sur le débourrement de pommiers, dans les conditions climatiques de plaines au sud du Maroc. Matériel et méthodes. Les expérimentations ont porté sur la variété 'Dorsett Golden' connue pour ses faibles besoins en froid. Un dispositif en bloc aléatoire complet avec quatre répétitions a été utilisé. Les produits testés ont été le Dormex appliqué soit seul à $(1,5,2,5$ et 4) \% du produit formulé, soit à $0,5 \%$ en association avec de l'huile minérale à ( 2 ou 4$) \%$, et l'acide gibbérellique à $460 \mathrm{mg} \cdot \mathrm{L}^{-1}$. Les arbres témoins ont été traités à l'eau. L'évaluation a porté sur le débourrement, la floraison, la nouaison, la croissance végétative et la production. Résultats. Les substances chimiques ont avancé la date de débourrement et en ont amélioré le taux de même que celui de floraison, et ils ont favorisé la croissance végétative. Le rendement et le calibre des fruits ont également été améliorés de manière significative. Conclusion. Le Dormex, seul ou combiné à de l'huile minérale, et l'acide gibbérellique ont avancé et regroupé le débourrement tout en améliorant le calibre et la production en fruits du pommier 'Dorsett Golden'. L'efficacité du mélange [Dormex + huile minérale] associée à son moindre coût conduisent à recommander ce produit pour pallier le manque en froid hivernal dans les zones de plaine du Maroc caractérisées par un hiver doux.

Maroc / Malus / levée de dormance / froid / cyanamide / gibberelline / floraison / fructification / rendement 


\section{Introduction}

Chez les rosacées fruitières, le froid constitue un facteur indispensable à la levée de dormance. Dans les plaines marocaines, son insuffisance limite la performance de variétés de pommier standard, telle 'Golden Delicious', et conduit à l'apparition d'une "dormance prolongée " caractéristique des régions à hiver doux. L'extension de la culture du pommier vers les zones de plaines et les zones de faible latitude pendant les années soixante-dix s'est retrouvée confrontée à ce phénomène physiologique aux conséquences graves. En effet, un manque en froid provoque un déséquilibre dans la croissance végétative et la fructification de l'arbre. Cette insuffisance peut se manifester entre autre par un taux de débourrement faible, un retard de débourrement des bourgeons latéraux, un débourrement anticipé des bourgeons terminaux, une inhibition du débourrement des bourgeons latéraux (effet de dominance apical), une insuffisance de la couverture foliaire et un rendement faible de fruits qui sont alors souvent de petit calibre [1]

De nombreuses recherches ont été entreprises dans différents pays pour mettre au point des techniques permettant d'atténuer les effets de cette dormance prolongée. Les travaux ont porté sur l'obtention de variétés à faibles besoins en froid ou bien sur l'utilisation de techniques culturales ou de produits chimiques susceptibles de stimuler la levée de dormance. C'est ainsi que de nombreuses variétés de pêcher et quelques variétés de pommier ont été créées dans le cadre de programmes d'hybridation pour étendre la culture de ces espèces à des zones dites à hiver doux. Pour le pommier, deux variétés 'Anna', et 'Dorsett Golden' ont connu une extension importante, notamment dans des zones de basse altitude et de faible latitude. L'introduction de ces variétés a permis l'extension de la culture du pommier à des zones du Maroc où il n'était pas cultivé auparavant. Toutefois, la performance de ces variétés censées répondre à ce problème est restée bien en deçà des attentes des producteurs.

Plusieurs produits chimiques, de nature différente et susceptibles d'influencer la levée de dormance des arbres fruitiers quand le froid est insuffisant ont été testés : le DNOC (dinitro-ortho-crésol), le nitrate de potassium $\left(\mathrm{KNO}_{3}\right)$, la thiourée, la benzyladénine (BA), la cyanamide hydrogène (Dormex, Calliope, Noguères, France), les cytokinines, l'éthrel, les gibbéréllines [2].

Le traitement des arbres fruitiers par des émulsions d'huiles minérales est le traitement qui a été le plus longtemps utilisé pour lever le repos végétatif dans les régions à hiver doux. Les facteurs dose et date sont alors très importants pour l'efficacité du traitement chimique car il y a des risques de phytotoxicité qui s'exprime surtout sur les organes floraux. Dans ce domaine, les espèces à noyau seraient beaucoup plus sensibles que les espèces à pépins dont le bourgeon floral est très protégé [3].

Les traitements précoces ont un effet forçant qui permet à l'arbre traité d'avoir une feuillaison et une floraison plus rapide que l'arbre témoin, cependant l'irrégularité dans le débourrement des bourgeons n'est pas éliminée alors que, dans le cas des traitements tardifs, le nombre de bourgeons qui s'ouvrent est groupé et augmenté [4]. L'utilisation de concentrations élevées d'huile [de $(2,4$ à 8,8$) \%$ ] et de faibles concentrations de $\operatorname{DNOC}(0,12 \%)$ a permis d'obtenir un meilleur taux d'ouverture des bourgeons végétatifs latéraux chez 'Golden Delicious' [5].

Dans la région du Sais au Maroc, la pulvérisation d'un mélange à (3 ou 5) \% d'huile de pétrole et $0,15 \%$ de DNOC sur des pommiers de la variété 'Golden Delicious' a augmenté le taux du débourrement, regroupé la floraison et la feuillaison, amélioré la croissance végétative et la qualité du fruit [6]. Les taux de nouaison, le calibre, le poids moyen de fruit et le rendement ont été également améliorés [7, 8]. L'amélioration du débourrement induite par ces produits a été plus importante pour les bourgeons à bois que pour des bourgeons floraux [6-8].

Des pulvérisations de Dormex à des concentrations de (1 à 5) \% ont accéléré et augmenté le pourcentage de débourrement de pommiers, pêchers, abricotiers et vigne [9-11]. Par ailleurs, la cyanamide a amélioré 
la qualité des fruits, augmenté la production et avancé la date de maturité de framboisiers [12]. L'efficacité de la cyanamide semble dépendre de la quantité de froid reçue par les plants avant le traitement [13]. Un test, dans la région du Gharb au Maroc, de l'effet du Dormex [à (2, 4 et 8) \% du produit formulé] sur la variété de pommier 'Golden Delicious' a montré que le produit était plus efficace lorsqu'il était appliqué $33 \mathrm{j}$ avant le débourrement naturel des bourgeons que lorsqu'il était appliqué 48 j avant cette date, sans qu'il n'y ait de différence notable entre les trois concentrations [14]. L'application de la cyanamide hydrogène a alors avancé, homogénéisé et amélioré le débourrement des bourgeons végétatifs et floraux. Cependant, le taux de nouaison et, par conséquent, le nombre de fruits sur les arbres traités ont étés inférieurs à ceux récoltés sur les arbres témoins.

Afin de mieux préciser le comportement " naturel" de la variété 'Dorsett Golden' connue pour ses faibles besoins en froid, nous avons mené une étude dans les conditions climatiques du sud du Maroc. Puis, nous avons étudié les effets du Dormex (seul et en association avec de l'huile de pétrole) et de l'acide gibbérellique sur la levée de dormance de cette variété.

\section{Matériel et méthodes}

\subsection{Caractéristiques de l'essai}

L'expérimentation a été menée dans un domaine privé de la région de Taroudant, au Sud du Maroc, sur des pommiers de la variété 'Dorsett Golden' greffés sur franc pied et plantés en 1994 à une distance de $5 \mathrm{~m} \times 3 \mathrm{~m}$, soit à une densité de 667 arbres $\cdot \mathrm{ha}^{-1}$, et conduits en gobelet. Les arbres ont été traités avec du Dormex ( $49 \%$ de cyanamide hydrogène en solution aqueuse stabilisée), de l'huile minérale et de l'acide gibbérellique. Sept traitements ont été appliqués le 11 décembre 1998. Ils se caractérisent comme suit :

- arbres témoins traités à l'eau (0\% Dormex),
- Dormex à $1,5 \%, 2,5 \%$ et $4 \%$ du produit formulé,

- Dormex à 0,5\% du produit formulé, combiné avec de l'huile minérale à $2 \%$ et $4 \%$,

- acide gibbérellique à $460 \mathrm{mg} \cdot \mathrm{L}^{-1}$.

Le dispositif expérimental a comporté des blocs aléatoires complets de quatre répétitions, l'arbre constituant l'unité expérimentale.

Les traitements appliqués à l'aide d'un pulvérisateur à dos de $20 \mathrm{~L}$ ont permis de mouiller uniformément le bois. Chaque arbre a reçu $3 \mathrm{~L}$ de solution.

\subsection{Observations et mensurations}

\subsubsection{Débourrement et floraison}

L'effet des produits testés pour leur efficacité à lever la dormance a été évalué sur le comportement de deux types de rameaux d'âges différents : rameaux de 1 an (notés $\mathrm{R}_{1}$ ) et rameaux de 2 ans (notés $\mathrm{R}_{2}$ ). Pour chaque traitement, quatre rameaux de chacun de ces deux types, choisis à hauteur d'homme et situés aux quatre points cardinaux de l'arbre pour tenir compte d'un éventuel effet "exposition", ont été marqués.

À partir du 14 janvier 1999, un comptage systématique hebdomadaire des bourgeons débourrés sur ces rameaux a permis de contrôler le débourrement. Les stades repères choisis ont été le stade " $\mathrm{C}$ " de Fleckinger pour les bourgeons à fleurs et le stade "début d'étalement des feuilles " pour les bourgeons à bois. Par ailleurs, tout bourgeon floral ayant atteint le stade $\mathrm{F}_{2}$ de Fleckinger a été considéré comme fleuri.

Les taux de débourrement, mesurés indépendamment sur bourgeons végétatifs et floraux, et les taux de floraison ont été calculés par les formules :

Taux de débourrement $(\%)=[$ nombre de bourgeons débourrés / nombre total de bourgeons) $\times 100$ ];

Taux de floraison $(\%)=[$ (nombre de bourgeons fleuris / nombre total de bourgeons) $\times 100$ ]. 


\subsubsection{Croissance végétative}

La croissance végétative des rameaux soumis aux différents traitements appliqués a été suivie par la mesure de la longueur de pousses terminales préalablement marquées dans la zone périphérique de la frondaison, à hauteur d'homme. Ces observations ont été réalisées tous les 10 jours, à partir du 15 mars 1999 et jusqu'à la fin du mois d'avril.

\subsubsection{Nouaison}

Le nombre de fleurs nouées a été compté sur les rameaux $\mathrm{R}_{1}$ et $\mathrm{R}_{2}$ en prenant comme repère le stade "I " de Fleckinger. Le taux de nouaison a été évalué par le rapport [(nombre de fleurs nouées / nombre total de fleurs sur le rameau) $\times 100$ ]

\subsubsection{Production et calibre}

Le nombre de fruits par arbre, leur poids et leur répartition en classes de calibres différents ont été déterminés à la récolte. Trois classes de calibre ont été considérées : diamètre du fruit $<63 \mathrm{~mm}$ (vrac), diamètre du fruit compris entre (63 et 74) mm, soit 22 à 28 fruits par plateau à alvéoles, et diamètre du fruit $>74 \mathrm{~mm}$, soit 18 fruits par plateau.

\subsubsection{Analyse statistique des données}

Les mesures exprimées sous forme de proportion ont été analysées après leur transformation angulaire $\left(\arcsin x^{1 / 2}\right)$ afin de stabiliser les variances.

Les données ont été soumises à une analyse de la variance effectuée avec le logiciel Statitcf. La séparation des moyennes a été réalisée à l'aide du test de Newman et Keuls.

\section{Résultats}

\subsection{Débourrement des bourgeons végétatifs}

\subsubsection{Sur rameaux de 1 an}

Quelle que soit la concentration de Dormex appliquée, le taux de débourrement des bourgeons végétatifs sur rameaux $R_{1}$ a été amélioré durant toute la période de débourrement par rapport aux rameaux témoins (tableau I). Les différences significatives imputables aux différentes concentrations du produit observées en début de saison se sont estompées avec le temps. Les concentrations les plus fortes ont provoqué les effets les plus marqués. L'augmentation du débourrement apportée par les différentes concentrations a varié entre (100 et 122) \% par rapport au témoin en fin de débourrement.

\subsubsection{Sur rameaux de 2 ans}

Comme pour les rameaux $\mathrm{R}_{1}$, le taux de débourrement des bourgeons végétatifs sur rameaux $R_{2}$ traités a été amélioré durant toute la période de débourrement par rapport aux rameaux témoins (tableau I). En début de débourrement, l'analyse de la variance a révélé l'existence d'une différence très hautement significative liée aux différents produits et concentrations utilisés. Toutefois, cette différence s'est estompée avec la progression de la campagne. Ainsi, à partir du 12 février, l'analyse a révélé que les concentrations du produit n'entraînaient pas de différence significative des taux de débourrement des bourgeons végétatifs qui variaient cependant de façon significative selon que les arbres avaient été traités ou non. L'amélioration du débourrement apportée par les traitements a varié entre (109 et 122) \% par rapport au témoin en fin de débourrement.

\subsubsection{Sur rameaux de 1 an et de 2 ans}

Quelle que soit la date d'observation, le Dormex à différentes concentrations a amélioré le débourrement moyen des bourgeons végétatifs sur l'ensemble des rameaux $\mathrm{R}_{1}$ et $\mathrm{R}_{2}$, par rapport aux rameaux non traités (tableau I). Les concentrations appliquées ont donc agi à la fois sur la précocité et sur le regroupement du débourrement. L'effet dose apparent au début de la campagne s'est estompé avec le temps. Ainsi, les arbres traités ont présenté des taux de débourrement finaux similaires indépendamment de la dose de produit. L'amélioration du débourrement global des bourgeons végétatifs apporté par les produits a varié entre (107 et 118) \% en fin de débourrement. 
Tableau I.

Effet de la cyanamide hydrogène (seule ou combinée à de l'huile minérale) et de l'acide gibbérellique, appliqués le 11/12/98, sur l'évolution du taux de débourrement (\%) des bourgeons végétatifs sur rameaux de 1 an $\left(R_{1}\right)$ ou 2 ans $\left(R_{2}\right)$, chez le pommier 'Dorsett Golden' cultivé dans les conditions climatiques de Taroudant au sud du Maroc.

\begin{tabular}{|c|c|c|c|c|c|c|c|c|c|c|c|c|c|c|c|}
\hline \multirow[t]{2}{*}{ Dormex (\%) } & \multicolumn{3}{|c|}{15 janvier } & \multicolumn{3}{|c|}{22 janvier } & \multicolumn{3}{|c|}{29 janvier } & \multicolumn{3}{|c|}{5 février } & \multicolumn{3}{|c|}{12 février } \\
\hline & $\mathrm{R}_{1}$ & $\mathrm{R}_{2}$ & $\mathrm{R}_{1}+\mathrm{R}_{2}$ & $\mathrm{R}_{1}$ & $\mathrm{R}_{2}$ & $\mathrm{R}_{1}+\mathrm{R}_{2}$ & $\mathrm{R}_{1}$ & $\mathrm{R}_{2}$ & $R_{1}+R_{2}$ & $\mathrm{R}_{1}$ & $\mathrm{R}_{2}$ & $R_{1}+R_{2}$ & $\mathrm{R}_{1}$ & $\mathrm{R}_{2}$ & $\mathrm{R}_{1}+\mathrm{R}_{2}$ \\
\hline 0 (témoin) & 0 & 0 & 0 & 09 & 10 & 10 & 33 & 32 & 32 & 37 & 39 & 38 & 45 & 45 & 45 \\
\hline 1,5 & 20 & 20 & 20 & 22 & 31 & 27 & 68 & 58 & 64 & 79 & 80 & 80 & 92 & 94 & 93 \\
\hline 2,5 & 26 & 34 & 30 & 37 & 62 & 48 & 69 & 87 & 80 & 87 & 95 & 91 & 98 & 100 & 99 \\
\hline 4 & 21 & 46 & 32 & 30 & 64 & 47 & 87 & 82 & 84 & 89 & 92 & 90 & 100 & 96 & 98 \\
\hline $0,5+2 \%$ huile & 11 & 14 & 12 & 33 & 27 & 30 & 62 & 53 & 60 & 100 & 88 & 94 & 100 & 98 & 99 \\
\hline $0,5+4 \%$ huile & 13 & 17 & 10 & 43 & 28 & 35 & 64 & 55 & 60 & 91 & 85 & 88 & 91 & 96 & 95 \\
\hline $460 \mathrm{mg} \cdot \mathrm{L}^{-1} \mathrm{AG}_{3}$ & 14 & 08 & 11 & 21 & 18 & 20 & 62 & 49 & 55 & 89 & 85 & 87 & 100 & 100 & 100 \\
\hline
\end{tabular}

\section{Tableau II.}

Effet de la cyanamide hydrogène (seule ou combinée à de l'huile minérale) et de l'acide gibbérellique, appliqués le 11/12/98, sur l'évolution du taux de débourrement (\%) des bourgeons floraux sur rameaux de 1 an $\left(R_{1}\right)$ ou 2 ans $\left(R_{2}\right)$, chez le pommier 'Dorsett Golden' cultivé dans les conditions climatiques de Taroudant au sud du Maroc.

\begin{tabular}{|c|c|c|c|c|c|c|c|c|c|c|c|c|c|c|c|}
\hline \multirow[t]{2}{*}{ Dormex (\%) } & \multicolumn{3}{|c|}{15 janvier } & \multicolumn{3}{|c|}{22 janvier } & \multicolumn{3}{|c|}{29 janvier } & \multicolumn{3}{|c|}{5 février } & \multicolumn{3}{|c|}{12 février } \\
\hline & $\mathrm{R}_{1}$ & $\mathrm{R}_{2}$ & $\mathrm{R}_{1}+\mathrm{R}_{2}$ & $\mathrm{R}_{1}$ & $\mathrm{R}_{2}$ & $\mathrm{R}_{1}+\mathrm{R}_{2}$ & $\mathrm{R}_{1}$ & $\mathrm{R}_{2}$ & $\mathrm{R}_{1}+\mathrm{R}_{2}$ & $\mathrm{R}_{1}$ & $\mathrm{R}_{2}$ & $\mathrm{R}_{1}+\mathrm{R}_{2}$ & $\mathrm{R}_{1}$ & $\mathrm{R}_{2}$ & $\mathrm{R}_{1}+\mathrm{R}_{2}$ \\
\hline 0 (témoin) & 0 & 0 & 0 & 5 & 7 & 8 & 15 & 24 & 24 & 26 & 42 & 35 & 41 & 47 & 45 \\
\hline 1,5 & 16 & 15 & 16 & 33 & 36 & 34 & 68 & 67 & 67 & 97 & 81 & 86 & 98 & 92 & 94 \\
\hline 2,5 & 25 & 32 & 30 & 44 & 56 & 50 & 67 & 85 & 78 & 90 & 98 & 94 & 96 & 100 & 100 \\
\hline 4 & 5 & 4 & 5 & 22 & 20 & 21 & 65 & 53 & 65 & 88 & 83 & 86 & 100 & 98 & 98 \\
\hline $0,5+2 \%$ huile & 11 & 18 & 15 & 22 & 43 & 32 & 65 & 74 & 70 & 87 & 98 & 92 & 98 & 99 & 98 \\
\hline $0,5+4 \%$ huile & 8 & 5 & 7 & 54 & 44 & 48 & 74 & 66 & 70 & 92 & 82 & 87 & 96 & 90 & 93 \\
\hline $460 \mathrm{mg} \cdot \mathrm{L}^{-1} \mathrm{AG}_{3}$ & 10 & 10 & 10 & 32 & 26 & 29 & 58 & 54 & 56 & 90 & 76 & 83 & 100 & 94 & 97 \\
\hline
\end{tabular}

\subsection{Débourrement des bourgeons floraux}

\subsubsection{Sur rameaux de 1 an}

Tous les produits testés vis-à-vis de leur effet sur la levée de dormance des bourgeons floraux ont amélioré de manière significative le débourrement de ces bourgeons sur rameaux $R_{1}$ par rapport aux rameaux témoins (tableau II). Les taux de débourrement finaux, au 12 février, ont été similaires pour les arbres traités, quel que soit le produit utilisé. L'amélioration du débourrement final des bourgeons floraux sur rameaux de 1 an imputable aux concentrations utilisées a varié entre (134 et 144) \% par rapport au témoin.

\subsubsection{Sur rameaux de 2 ans}

Quelle que soit la date d'observation considérée, la solution appliquée a amélioré le débourrement des bourgeons floraux sur les rameaux de 2 ans (tableau II). L'effet produit ou dose ne s'est manifesté qu'au début de la phase de débourrement et s'est estompé par la suite. Les arbres traités ont eu des taux de débourrement similaires et très nettement supérieurs à celui enregistré sur les arbres témoins. L'amélioration du débourrement final apportée par les traitements a varié entre (96 et 113) \%.

\subsubsection{Sur rameaux de 1 an et 2 ans}

Globalement, par rapport aux arbres témoins non traités, le débourrement des 
Figure 1.

Effets de la cyanamide hydrogène, seule ou associée à de l'huile minérale, et de l'acide gibbérellique appliqués le 11/12/98 sur la floraison du pommier 'Dorsett Golden' cultivé dans les conditions climatiques de Taroudant au sud du Maroc.

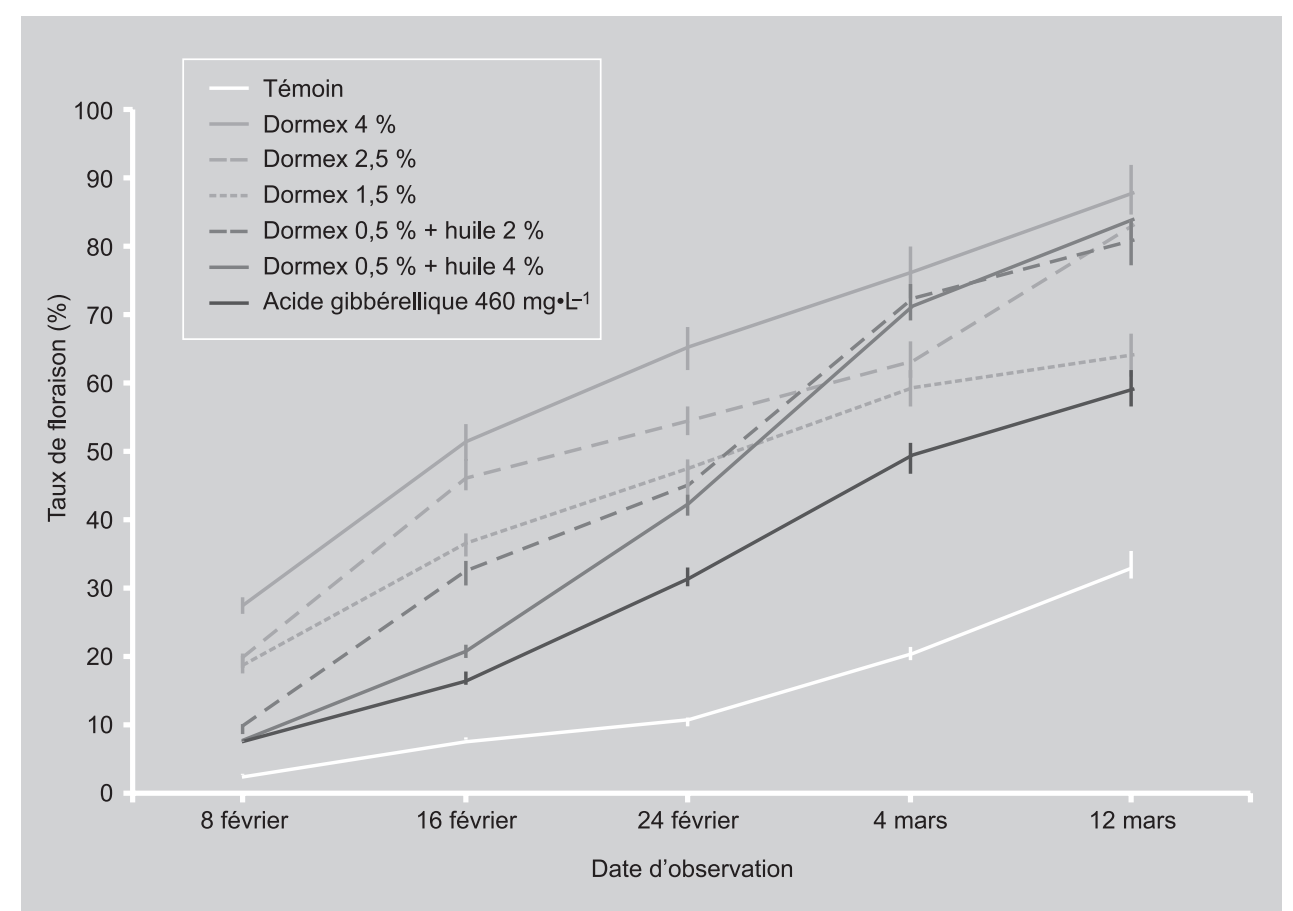

bourgeons floraux sur l'ensemble des rameaux de 1 an et 2 ans a été amélioré de manière significative par le Dormex seul ou combiné avec de l'huile minérale, ainsi que par l'acide gibbérellique (tableau II). L'amélioration du débourrement final des bourgeons floraux imputable aux substances chimiques a varié de (111 à 122) \% par rapport au témoin.

\subsection{Précocité du débourrement}

La précocité du débourrement a été évaluée en comparant la date à laquelle les arbres témoins non traités avaient atteint leur taux de débourrement maximal avec les dates auxquelles les arbres traités avaient atteint le même niveau de débourrement. Ainsi, il a été observé que l'application des substances chimiques permettait d'avancer la date de débourrement aussi bien pour les bourgeons végétatifs que pour les bourgeons floraux. Cet avancement a varié de (21 à 28) jours pour les bourgeons végétatifs et de (20 à 24) jours pour les bourgeons floraux. La précocité du débourrement et l'amélioration de son taux constatée précédemment permettraient une formation rapide du fruit et le développement d'une feuillaison adéquate susceptible d'assurer une production normale de pommes d'un calibre commercialisable.

\subsection{Floraison}

\subsection{1. Évolution du taux de floraison}

L'application de Dormex et d'acide gibbérellique a permis d'augmenter le taux de floraison des arbres traités [entre (60 et 89) \%, figure 1] par rapport aux arbres témoins non traités dont le taux n'a pas dépassé $34 \%$. Cela correspond à une amélioration de (76 à 162) \% par rapport au témoin. En absence de traitement, il y aurait donc eu un avortement d'une proportion importante de bourgeons floraux. Ce désordre physiologique est connu pour être l'un des symptômes caractéristiques d'une insuffisance en froid. Par ailleurs, la floraison a été beaucoup plus étalée sur les arbres témoins que sur les arbres traités. Le regroupement de la floraison observé sur ces deniers arbres serait apte à réunir des conditions favorables à une bonne nouaison propice à la formation de fruits capables de poursuivre leur développement jusqu'à maturité. 


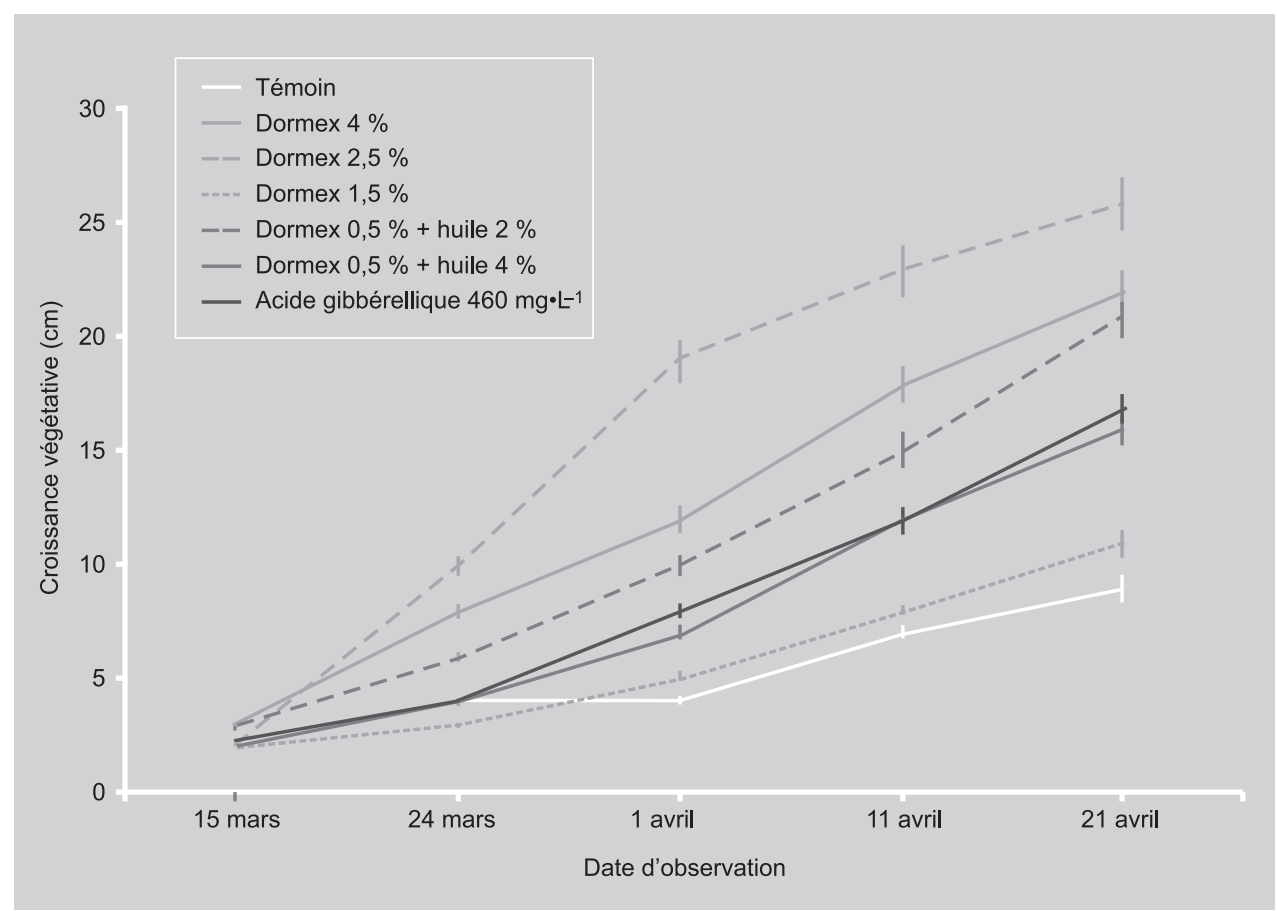

L'addition d'huile minérale a amélioré l'efficacité de la cyanamide hydrogène. En effet, le Dormex à 0,5\% appliqué seul aurait un effet limité sur le débourrement et la floraison. En revanche, l'adjonction d'huile minérale à $2 \%$ a rendu cette concentration aussi efficace qu'une concentration de Dormex à $2,5 \%$, voire à $4 \%$. De tels traitements combinés seraient intéressants en raison de leur efficacité et du moindre coût de l'huile minérale par rapport à celui du produit chimique de traitement.

\subsubsection{Précocité de floraison}

L'application de produits chimiques, cynamide hydrogène et acide gibbérellique, a permis d'avancer la date de pleine floraison de (13 et 27) jours (figure 1). L'application de Dormex a donc agi sur la date de floraison qui a eu lieu plus tôt sur les arbres ainsi traités que sur les plants témoins, et ce quelles que soient les doses et les substances appliquées.

\subsection{Nouaison}

Le taux de nouaison semble être affecté de manière négative par le Dormex. En effet, l'analyse de la variance a révélé l'existence d'une différence significative entre les taux de nouaison des arbres traités et celui des arbres témoins. Le taux moyen de nouaison enregistré sur ces derniers (56\%) est significativement supérieur à celui observé sur les arbres traités (non différents entre eux) qui a varié entre (32 et 39) \%. Toutefois, la nouaison exprimée en nombre de fruits formés par arbre s'est retrouvée nettement améliorée sur les arbres traités en raison de l'amélioration de leurs taux de floraison induite par les substances chimiques. L'effet négatif du Dormex sur le taux de nouaison ne s'est pas manifesté lorsqu'il a été appliqué à $0,5 \%$ en combinaison avec l'huile minérale.

\subsection{Croissance végétative}

Le Dormex utilisé seul ou additionné d'huile minérale ainsi que l'acide gibbérellique ont amélioré la croissance des pousses terminales (figure 2). Le traitement au Dormex à $2,5 \%$ a induit la plus forte croissance $(26 \mathrm{~cm})$, puis celui à $4 \%$ a permis une croissance légèrement moindre $(22 \mathrm{~cm})$ alors que la plus faible concentration de Dormex à 1,5\% (11 cm) n'a pas
Figure 2.

Effets de la cyanamide hydrogène, seule ou associée à de l'huile minérale, et de l'acide gibbérellique appliqués le $11 / 12 / 98$ sur la croissance végétative du pommier 'Dorsett Golden' cultivé dans les conditions climatiques de Taroudant au sud du Maroc. 
Figure 3.

Effets de la cyanamide hydrogène, seule ou associée à de l'huile minérale, et de l'acide gibbérellique appliqués le 11/12/98 sur la croissance du fruit du pommier 'Dorsett Golden' cultivé dans les conditions climatiques de Taroudant au sud du Maroc.

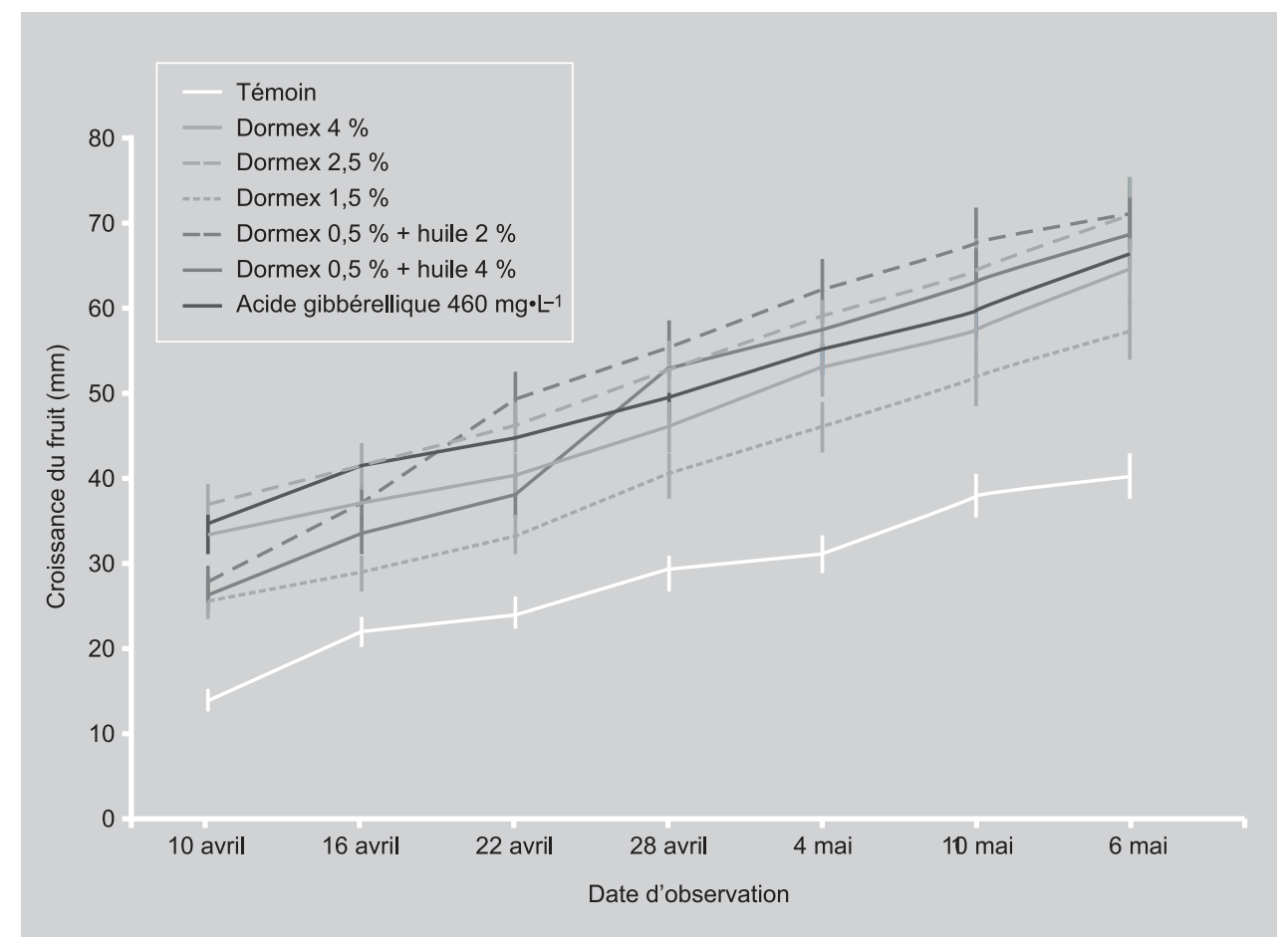

donné de résultats significativement différent du témoin $(9 \mathrm{~cm})$.

\subsection{Fruit et rendement}

\subsubsection{Croissance du fruit}

Tous les traitements chimiques testés ont amélioré la vitesse de croissance du fruit avec une légère supériorité pour les concentrations élevées de Dormex, les traitements combinés [huile + Dormex] et l'acide gibbérellique (figure 3). Cela a conduit à l'obtention d'un fruit de calibre supérieur chez les arbres traités par rapport à celui obtenu sur les arbres témoins. Cette amélioration de la croissance du fruit résulterait de la précocité du débourrement et de la floraison, ainsi que d'une meilleure croissance végétative qui seraient induites par l'utilisation des substances chimiques.

\subsubsection{Nombre de fruits, rendement et calibre}

Les arbres traités ont présenté un nombre de fruits, un calibre et un rendement par arbre supérieurs à ceux du témoin (tableau III).
L'amélioration du rendement des pommiers traités par rapport aux arbres témoins a varié entre $35 \%$ pour l'utilisation de Dormex à $1,5 \%$ et $75 \%$ pour l'emploi de Dormex à $0,5 \%$ additionné d'huile minérale à $2 \%$. Ce résultat est dû à l'augmentation du nombre de fruits par arbre et à l'amélioration du calibre et du poids du fruit.

\section{Discussion et conclusion}

Le Dormex, seul ou additionné d'huile de pétrole, ainsi que l'acide gibbérellique ont amélioré de manière significative les taux de débourrement des bourgeons végétatifs et floraux sur les rameaux de 1 an et de 2 ans de pommiers de la variété 'Dorsett Golden'. Pour les deux types de bois, l'amélioration du débourrement induite par les traitements a été de même importance pour les bourgeons végétatifs et pour les bourgeons floraux, alors que, sur 'Golden Delicious', l'amélioration du débourrement induite par le DNOC a été trouvée plus importante pour les bourgeons végétatifs que pour les bourgeons floraux [8]. 
Tableau III.

Effet de la cyanamide hydrogène (seule ou combinée à de l'huile minérale) et de l'acide gibbérellique, appliqués le 11/12/98, sur le rendement, le nombre de fruits par arbre et le calibre du fruit du pommier 'Dorsett Golden' dans les conditions climatiques de Taroudant au sud du Maroc.

\begin{tabular}{|c|c|c|c|c|c|c|c|c|c|}
\hline \multirow[t]{3}{*}{$\begin{array}{l}\text { Dormex } \\
(\%)\end{array}$} & \multirow[t]{3}{*}{$\begin{array}{l}\text { Rendement } \\
\left(\mathrm{t} \cdot \mathrm{ha}^{-1}\right)\end{array}$} & \multirow[t]{3}{*}{$\mathrm{kg} \cdot \operatorname{arbre}^{-1}$} & \multirow{3}{*}{$\begin{array}{l}\text { Nombre } \\
\text { de fruits } \\
\text { par arbre }\end{array}$} & \multicolumn{6}{|c|}{$\begin{array}{l}\text { Répartition de fruits } \\
\text { par classe de calibre par arbre }\end{array}$} \\
\hline & & & & \multicolumn{2}{|c|}{$<63 \mathrm{~mm}$} & \multicolumn{2}{|c|}{$\mathrm{De}(63$ à 74$) \mathrm{mm}$} & \multicolumn{2}{|c|}{$>74 \mathrm{~mm}$} \\
\hline & & & & (\%) & $\mathrm{kg}$ & $(\%)$ & $\mathrm{kg}$ & $(\%)$ & $\mathrm{kg}$ \\
\hline Témoin & 10 & 15 & 143 & 83 & 11,4 & 16 & 3,4 & 1 & 0,2 \\
\hline 1,5 & 13,5 & 20 & 179 & 70 & 11,6 & 26 & 7,0 & 4 & 1,4 \\
\hline 2,5 & 16 & 24 & 187 & 59 & 12,0 & 34 & 9,3 & 7 & 2,8 \\
\hline 4 & 16 & 24 & 183 & 62 & 12,6 & 28 & 7,7 & 10 & 3,6 \\
\hline $0,5+2 \%$ huile & 17,5 & 26 & 191 & 51 & 11,8 & 41 & 11,2 & 8 & 3,0 \\
\hline $0,5+4 \%$ huile & 14,5 & 22 & 182 & 66 & 12,2 & 27 & 7,4 & 7 & 2,4 \\
\hline $460 \mathrm{mg} \cdot \mathrm{L}^{-1} \mathrm{AG}_{3}$ & 13,5 & 20 & 174 & 69 & 12,2 & 29 & 7,3 & 2 & 0,5 \\
\hline
\end{tabular}

Outre l'amélioration des taux le débourrement, les traitements appliqués ont permis d'avancer le débourrement de l'ordre de 3 semaines et de le regrouper dans le temps. Cette précocité du débourrement s'est répercutée sur la date de floraison qui a été avancée à son tour. Les produits utilisés ont également amélioré la proportion des bourgeons floraux ayant atteint la pleine floraison, qui de $34 \%$ observés chez le témoin est passée à $60 \%$ lors de l'emploi d'acide gibbérellique et $65 \%$ lors du traitement au Dormex. Avec les autres applications, le taux de bourgeons floraux parvenus à floraison a dépassé le seuil de 80 \%. L'augmentation de floraison a donc finalement été de (76 à 162) \%.

Le taux de nouaison a été influencé de manière négative par le Dormex appliqué seul. Cet effet n'a pas été observé avec les autres substances chimiques. Cependant, la nouaison exprimée en terme de nombre de fruits par unité de production (données non publiées) ou par arbre a été supérieure sur les arbres traités. L'augmentation du nombre de fruits par arbre serait la conséquence de l'amélioration de l'intensité de floraison et de son regroupement. Cette différence importante du nombre de fruits par arbre entre les arbres traités et les arbres témoins a été répercutée sur la production. En effet, les arbres non traités ont présenté un rendement nettement inférieur à celui enregistré pour les arbres traités.

Par rapport aux arbres témoins, tous les arbres traités ont présenté des rendements améliorés. Cette amélioration a varié entre $35 \%$ pour une application de Dormex à $1,5 \%$ ou d'acide gibbérellique et $75 \%$ pour le traitement au Dormex à $0,5 \%$ additionné de $2 \%$ d'huile. Avec ces produits utilisés pour lever la dormance de la variété de pommier 'Dorsett Golden', le nombre de fruits par arbre, le calibre de ces fruits et leur poids peuvent donc être améliorés. Le traitement [Dormex à 0,5\% + huile minérale à $2 \%$ ] peut finalement être recommandé pour une utilisation en verger de pommiers dans le sud du Maroc, car il allie efficacité et moindre coût.

\section{Références}

[1] Petri J.L., L'interruption de dormance de pommier, BASF informations agricoles 2 (1989) 17-20.

[2] Erez A., Lavee S., The effect of climatic conditions on dormancy development of peach buds, J. Am. Soc. Hortic. Sci. 96 (1971) 711-714.

[3] Erez A., Chemical control of bud break, HortScience 22 (1987) 1240-1243. 
[4] Diaz D.H., Alvarez A., Sandoval J., Cultural and chemical practices to induce uniform bud break of peach and apple under warm climates in Mexico, Acta Hortic. 199 (1987) 99-136.

[5] Erez A., Zuret A., Breaking the rest of apple buds by narrow-distillation-range oil and dinitro-o-cresol, Sci. Hortic.-Amsterdam 14 (1981) 47-54.

[6] Mahhou A., Taoura M., Walali Loudiyi D., Effects of dinitro-ortho-cresol (DNOC) and oil on bud break of 'Golden Delicious' apple in the Saiss Area of Morocco, Actes Inst. Agron. Vét. 10 (3) (1990) 27-32.

[7] Mahhou A., Hakam M., Effets du mélange dinitro-ortho-cresol-huile de pétrole sur la levée de dormance du pommier (Malus domestica L.) dans la région du Gharb, Actes Inst. Agron. Vét. 15 (4) (1995) 37-42.

[8] Mahhou A., El Phil A., Effets du dinitro-orthocrésol (DNOC) sur la levée de dormance du pommier 'Golden Delicious' cultivé en plaine marocaine, Fruits 55 (2001) 367-376.
[9] Williams W.T., Tax Tzoc B.A., Preliminary observations of the effects of hydrogen cyanamid on breaking dormancy and harvest of apples in Guatemala, Acta Hortic. 279 (1990) 399-408.

[10] George A.P., Loyd J.P., Nissen R.J., Effects of hydrogen cyanamid, paclobutrazol and pruning date on dormancy release of the low chill peach cultivar 'Flordaprince' in subtropical Australia, Aust. J. Exp. Agr. 32 (1992) 89-95.

[11] Finetto G.A., The effect of hydrogen cyanamid on breaking endo-dormancy of midchilling apple cultivars in Yemen during two years, Acta Hortic. 329 (1993) 268-270.

[12] Snir I., Chemical dormancy breaking of red raspberry, HortScience 18 (1983) 710-713.

[13] Snir l., Erez A., Bloom advancement in sweet cherry by hydrogen cyanamid, Fruit Varieties J. 42 (1988) 120-122.

[14] Mahhou A., Hakam M., Effets de la cyanamide d'hydrogène sur la levée de dormance du pommier (Malus domestica Borkh.), Actes Inst. Agron. Vét. 13 (4) (1993) 13-19.

\section{Efectos de la cianamida de hidrógeno en la ruptura de la latencia del manzano 'Dorsett Golden' en el sur de Marruecos.}

Resumen - Introducción. El insuficiente frío invernal en las zonas de llanura en Marruecos constituye un factor limitante para la producción del manzano. Hemos evaluado los efectos de la cianamida de hidrógeno (Dormex), sola o con aceite mineral y ácido giberélico, en el desborre de manzanos, en las condiciones climáticas de las llanuras del sur de Marruecos. Material y métodos. Los experimentos se efectuaron con la variedad 'Dorsett Golden' conocida por sus escasos requerimientos de frío. Se empleó un diseño de bloque completo al azar con cuatro repeticiones. Se probó el Dormex aplicado sin mezcla al (1,5, 2,5 y 4)\% del producto formulado o bien al $0,5 \%$ asociado con aceite mineral al (2 ó 4)\% y ácido giberélico a $460 \mathrm{mg} \cdot \mathrm{L}^{-1}$. Los testigos no recibieron ningún tratamiento. La evaluación comprendió desborre, floración, cuajado, crecimiento vegetativo y producción. Resultados. Las sustancias químicas adelantaron la fecha de desborre mejorando su tasa al igual que la de floración; también favorecieron el crecimiento vegetativo. El rendimiento y el calibre de los frutos también mejoraron significativamente. Conclusión. El Dormex, solo o combinado con aceite mineral, y ácido giberélico adelantaron y agruparon el desborre mejorando, al mismo tiempo, el calibre y la producción frutal del manzano 'Dorsett Golden'. La eficacia de la mezcla [Dormex + aceite mineral] unida a su menor costo hacen que se recomiende este producto para paliar la escasez de frío invernal en las zonas llanas de Marruecos caracterizadas por un invierno suave.

\section{Marruecos / Malus / salida de la latencia / frío / cianamidas / ácido giberélico /} floración / fructificación / rendimiento 